\title{
Effect of increased fruit and vegetable intake on oxidative stress and airway inflammation in patients with moderate to severe chronic obstructive pulmonary disease
}

\author{
F. R. Baldrick ${ }^{1}$, J. S. Elborn ${ }^{2}$, J. V. Woodside ${ }^{1}$, K. Treacy ${ }^{2}$, J. Bradley ${ }^{2}$, B. Schock ${ }^{3}$, M. Ennis ${ }^{3}$, \\ I. S. Young ${ }^{1}$ and M. C. McKinley ${ }^{1}$ \\ ${ }^{1}$ Centre for Public Health, Queen's University Belfast, BT12 6BJ, UK, ${ }^{2}$ Respiratory Medicine Group, Belfast City Hospital, \\ BT9 7AB, UK and ${ }^{3}$ Respiratory Medicine Research Group, Queen's University, Belfast BT12 6BN, UK
}

Chronic obstructive pulmonary disease (COPD) is predicted to become the third most common cause of death by $2020^{(1)}$. Dietary constituents, such as fruits and vegetables (FV), possess anti-inflammatory and antioxidant properties and, hence, are of interest with respect to the pathogenesis of COPD. We conducted a pilot randomised controlled trial to examine the effect of increased consumption of FV on oxidative stress and airway inflammation in people with moderate to severe COPD.

Eighty one participants (62.2 (SD 8.7) years, BMI 27.7 (SD 5.8) $\mathrm{kg} / \mathrm{m}^{2}$ ) with moderate to severe COPD and a habitually low FV intake (1.5 (SD 0.7) portions/d) were randomized to either a low ( $\leq 2$ portions/d) or high ( $\geq 5$ portions/d) FV diet for 12 weeks; $n=75$ completed the intervention. All FV were provided and consumed in a free-living situation. Self-reported FV intake (diet history interviews) combined with a panel of biomarkers of FV intake were used to assess compliance to the diet. A number of markers of airway and systemic inflammation and oxidative stress were measured.

As detailed in the table below, self-reported FV intake and biochemical markers of FV intake both indicated good compliance with the intervention. Despite participants' adherence to the study protocol, there were no significant differences between the low-FV group and the high-FV group for the markers of oxidative stress or inflammation.

\begin{tabular}{|c|c|c|c|c|c|}
\hline \multirow[b]{2}{*}{ Variable } & \multicolumn{2}{|c|}{ Low-FV diet $\left(n=37_{\max }\right)$} & \multicolumn{2}{|c|}{ High-FV diet $\left(n=38_{\max }\right)$} & \multirow[b]{2}{*}{$P^{3}$} \\
\hline & Week $0^{1}$ & Week $12^{2}$ & Week 0 & Week 12 & \\
\hline FVintake (portions/d) & $1.4(0.6)^{4}$ & $1.9(0.5)^{* * *}$ & $1.5(0.7)$ & $6.1(1.8)^{* * *}$ & $<0.001 * * *$ \\
\hline Vitamin $C(\mu \mathrm{mol} / \mathrm{l})$ & $18.8(9.8-46.2)^{5}$ & $25.3(13.8-47.9) *$ & $28.1(19.2-48.9)$ & $43.4(36.8-64.5)^{* *}$ & 0.436 \\
\hline Lutein $(\mu \mathrm{mol} / \mathrm{l})$ & $0.14(0.10-0.21)$ & $0.16(0.12-0.22)^{*}$ & $0.15(0.11-0.21)$ & $0.19(0.14-0.25) * * *$ & 0.076 \\
\hline Zeaxanthm $(\mu \mathrm{mol} / \mathrm{l})$ & $0.03(0.02-0.03)$ & $0.03(0.02-0.04)$ & $0.02(0.02-0.03)$ & $0.03(0.02-0.04)^{* * *}$ & $0.034 *$ \\
\hline 3-cryptoxanthin $(\mu \mathrm{mol} / \mathrm{l})$ & $0.03(0.03-0.05)$ & $0.04(0.03-0.06)^{* *}$ & $0.04(0.03-0.06)$ & $0.07(0.04-0.11)^{* * *}$ & $0.015^{*}$ \\
\hline IL-8 (ng/ml) & $9.0(4.5-18.5)$ & $7.7(3.9-13.5)$ & $11.7(5.4-19.2)$ & $10.00(4.5-15.9)$ & 0.992 \\
\hline MPO (ng/ml) & $956(467-1961)$ & $947(491-1981)$ & $1218(590-2211)$ & $1384(784-1828)$ & 0.5 \\
\hline $\mathrm{NE}(\mathrm{mg} / \mathrm{ml})$ & $4.93(0.18-17.20)$ & $2.92(0.68-4.61)$ & $2.94(0.77-11.16)$ & $2.33(0.64-7.72)$ & 0.621 \\
\hline CRP (mg/l) & $4.15(1.60-7.98)$ & $3.39(1.89-6.21)$ & $3.38(1.66-5.74)$ & $4.09(1.98-5.99)$ & 0.116 \\
\hline CRP (pg/ml) & $6.0(3.0-38.6)$ & $6.4(3.0-30.1)$ & $13.9(3.0-95.6)$ & $8.3(3.0-54.3)$ & 0.258 \\
\hline 8-iso (nм/mm creatinine) & $1.54(1.20-1.97)$ & $1.41(1.04-1.74)$ & $1.76(1.20-2.40)$ & $1.65(1.20-2.27)$ & 0.806 \\
\hline 8-iso (ng/ml) & $1.84(1.20-3.21)$ & $1.70(1.32-2.43)$ & $1.44(1.12-3.24)$ & $1.83(1.20-2.63)$ & 0.334 \\
\hline
\end{tabular}

${ }^{1}$ Baseline values (before diet) did not differ significantly between groups, $P>0.05$ (independent samples $t$-test)

${ }^{2}$ Within group comparisons analysed by paired samples $t$-test, significantly different from baseline $(* P<0.05, * * P<0.01, * * * P<0.001)$.

${ }^{3}$ Analysed by independent samples $t$-test, significantly different $(* P<0.05)$.

${ }^{4}$ Values are mean (SD).

${ }^{5}$ Values are geometric mean (interquartile range). IL-8, interleukin 8; MPO, myeloperoxidase; NE, neutrophil elastase; CRP, C-reactive protein; 8-iso, 8-isoprostane.

Patients with moderate to severe COPD complied with an intervention to increase fruit and vegetable intake; however, this increased intake had no significant effect on airway and systemic inflammation and oxidative stress.

1. Lopez AD \& Murray CC (1998) Nat Med 4, 1241-1243. 